\title{
Clinical Trial and Preparation of Amniotic Membrane as Dura Mater Artificial
}

\author{
*Asra Al Fauzi, Adanti Wido Paramadini \\ Department of Neurosurgery \\ Airlangga University, Dr. Soetomo General Hospital \\ Surabaya, Indonesia \\ *asrafauzi@yahoo.com \\ danti.paramadini@gmail.com
}

\author{
Taufiq Fatchur Rochman \\ Department of Neurosurgery \\ Mulawarman University, A. Wahab Syahranie General \\ Hospital \\ Surabaya, Indonesia \\ taufiq.fatchur@gmail.com
}

\begin{abstract}
Duraplasty is a common procedure while human dura mater cannot be sutured during surgery and required a dural graft. Some material has been studied as dura mater substitute. One of those materials is amniotic membrane that used frequently in the surgical procedure. A clinical experimental study was performed to analyze the healing of dural in 8 patients at Dr. Soetomo General Hospital Surabaya, Indonesia. The amniotic membrane allograft, had been processed and folded in $\mathbf{1 6}$ layers. The membrane was then sutured to cover the dural defect. Ten weeks later, the patient underwent cranioplasty and CSF leakage evaluated by checking the edge of dural. The fibrocyte infiltration was examined histologically. Based on the analysis, the amniotic membrane showed great potential as dura mater artificial.
\end{abstract}

Key Words-Duraplasty; amniotic membrane; dura mater artificial

\section{INTRODUCTION}

Study of dura mater grafting has been conducted since 1890 with various material, such as autologous, metal sheet, body tissue and biosynthetic material. Dural graft should be completed with some properties such as inert, nontoxic, noncarcinogenic, water-resistant and able to maintain the stitches without sticking to the cortex. The material should be sterile, inexpensive and easy to obtain [1].

Dura graft or dura mater artificial being the important part of duraplasty procedure. It should be executed due to the following conditions: 1) removed part of dura mater 2) dura mater fistula repair or 3) enlargement of intracranial compartment. This case required watertight closure to maintain the stitches without sticking to the cortex.

Synthetic graft material often rejected by the patient's body because of local tissue inflammatory reaction. It was remarked by irritation of cortex underneath the brain, meningitis, excessive scar tissue formation and bleeding [2]. Another way to substitute dura mater was using cadaveric human dura, but it was not used anymore due to infection of carried prion disease, such as Creutzfeldt-Jakob [3]. However, it also required more excision.

Amniotic membrane has been used in surgery field for a long time, particularly in plastic and eye surgery. It has been applied because of its ability to stimulate epithelization process, inhibit excessive fibrosis, inflammation and angiogenesis, decrease immune response, and providing antimicrobial and antiviral effects. Amnion also effective to accelerate wound healing process, because it moisten the wound surface [4].

A study conducted by Tomita (2012) stated that amniotic membrane was effective to be dural graft during skull base surgery. There was no, tissue rejection, infection and CSF leakage.

During this research, we proceed the membrane manufacture thoroughly. This study was aimed to obtain the analyzatio of amniotic membrane as dura mater artificial based on clinical and histological review.

\section{METHODS}

This study approved legal/ethical clearance from the local medical research ethics committees of Dr. Soetomo General Hospital, Surabaya, Indonesia, following the regulatory guidelines of the country. Moreover, a research permit was obtained from the Research and Development Department of East Java Province, Indonesia. Informed consent documents, details of the medical treatment and other necessary approval documents were delivered to all patients involved in the study. Informed consent was obtained from all the patients who were included in this study.

\section{Inclusion Criteria \\ 1. Age 15 to 60 years \\ 2. Moderate head injury \\ 3. Severe head injury \\ 4. Spontaneous Intra Cerebral Hemorrhage}

Exclusion Criteria

1. Malnutrition

2. Diabetes mellitus

3. Infection 


\section{A. Tools and Materials}

Duraplasty with amniotic membrane used basic tools of craniotomy and basic tools of general anasthesia. Microscope was used for histological examination. The study materials was 16 layers amniotic membrane allograft with size of $10 \mathrm{~cm}$ $\mathrm{x} 10 \mathrm{~cm}$.

\section{B. Preparation of Amniotic Membrane}

Fresh placenta was obtained from newborn baby. It was collected aseptically in childbirth operating room. Meconium contaminated placenta was excluded, then the remaining placenta was then placed in sterile container. $\mathrm{NaCl}$ and antibiotic solution would be added whenever necessary. The amniotic membrane was then separated from the placenta and washed with physiological $\mathrm{NaCl}$ solution. It was stored at $4^{0} \mathrm{C}$ temperature. The sample could not be stored longer than 4 days before processing.

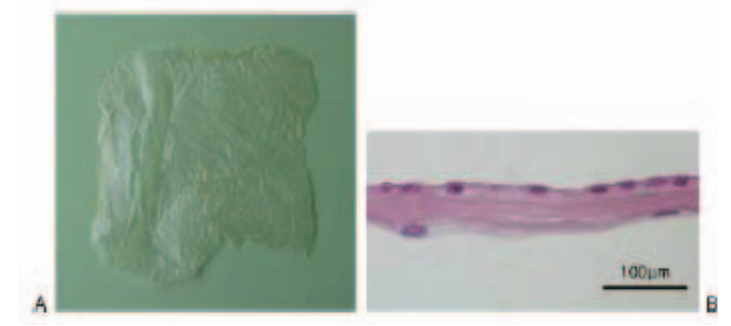

Fig. 1, Amniotic Membran (Tomita T, 2012)

Manufacture of amniotic membrane was performed under sterile conditions, and each step of the process was written on the log books. In the end of preparation, tissue samples were taken from remaining leaching solution of $\mathrm{NaCl}$. The samples would be used for microbiological examination before packaging process.

Amniotic membrane was soaked in $0.05 \%$ sodium hypochlorite for about 10 minutes, therefore $\mathrm{pH}$ solution could be neutral. The membrane was placed in a water bath shaker filled with sterile physiological $\mathrm{NaCl}$ solution at room temperature. The solution was then replaced periodically every 15 minutes for 10 times. A few drops of solution also taken for microbiological examination.

The amnion membrane was washed and placed on sterile gauze pad and cut into the efficient size for application. To keep sterile, the process was performed in the laminar air-flow cabinet. The membrane was store in a deep freezer for freeze drying process. Freeze dried was run for 6 hours until the water reached $6-7 \%$. The packaging process took place in the laminar air-flow cabinet using three layers of polyethylene plastic. Vacuum sealer was used for sealing process.

\section{Clinical Study}

After obtaining legal approval to do surgery, decompressive craniotomy was performed under general anesthesia. The average size of the craniotomy had to be at least $10 \times 5 \mathrm{~cm}$ then duraplasty was completed using amniotic membrane. Graft was then stitched together on the edge of dura using silk thread 4/0. The periosteum, muscle and scalp closed layer by layer.

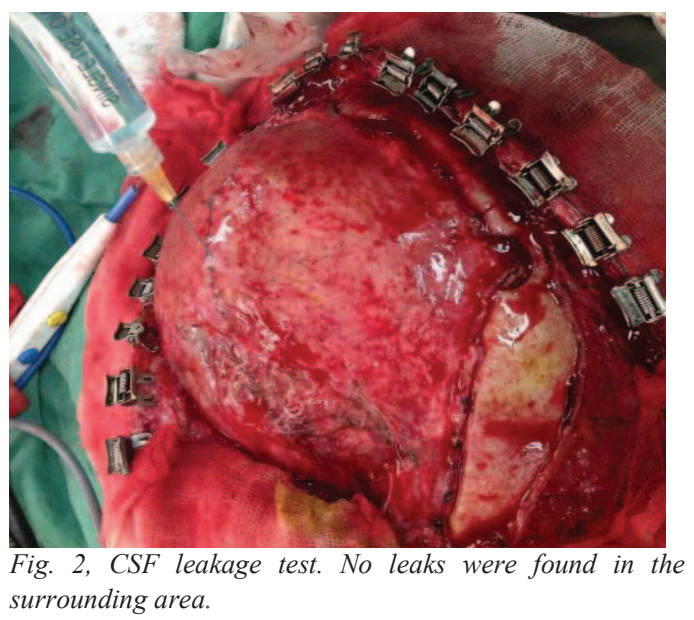

After more than four weeks, cranioplasty was performed using a skull bone autograft. The incision and dissection of the scalp, muscle, and periosteum were applied layer by layer to identified the dura and the graft. Povidoneiodine was attached to the dura and the graft. Ten millimeters of isotonic saline solution was injected into the subdural cavity using a $26 \mathrm{G}$ needle. CSF leakage was then checked on the edge of the dura-graft stitching. The disappearance of povidone-iodine swabs on the dura mater and the graft indicated CSF leakage. Once the checking was done, the dura mater and graft were flushed with sterile isotonic saline. The sample for histological analysis was obtained by cutting the edge of the dural graft suture into a size of $0.5 \times 0.5 \mathrm{~cm}$. Histological specimens were fixed with formalin. The edge of the defect and dural graft were primarily stitched using silk thread 4/0. Autograft cranioplasty was performed using miniplate-screw fixation. The periosteum, muscle, and scalp were then closed layer by layer.

\section{Histological Analysis}

The pieces of the dural graft edge were fixed in formalin and HE stained. The histological sample was analyzed by a microscope using a magnification of $40 \mathrm{x}$ and then evaluated for fibrocyte infiltration thickness at the edges of the dural graft.

\section{RESULTS AND DISCUSSION}

The use of amniotic membrane graft as a cover material for dural defects in duraplasty neurosurgical operations is a new alternative. Previous amniotic membrane graft studies with human subjects for duraplasty showed no tissue rejection, infection, or CSF leakage[5]. It can be showed from the results that no infection was found. 
We examined the watertight effect of amniotic membrane graft by making $0.9 \% \mathrm{NaCl}$ fluid injections into the subdural cavity. The CSF leakage inspection showed results $0 \%$ for all patients. It means there are no CSF leakage occurred in the dural graft extension.

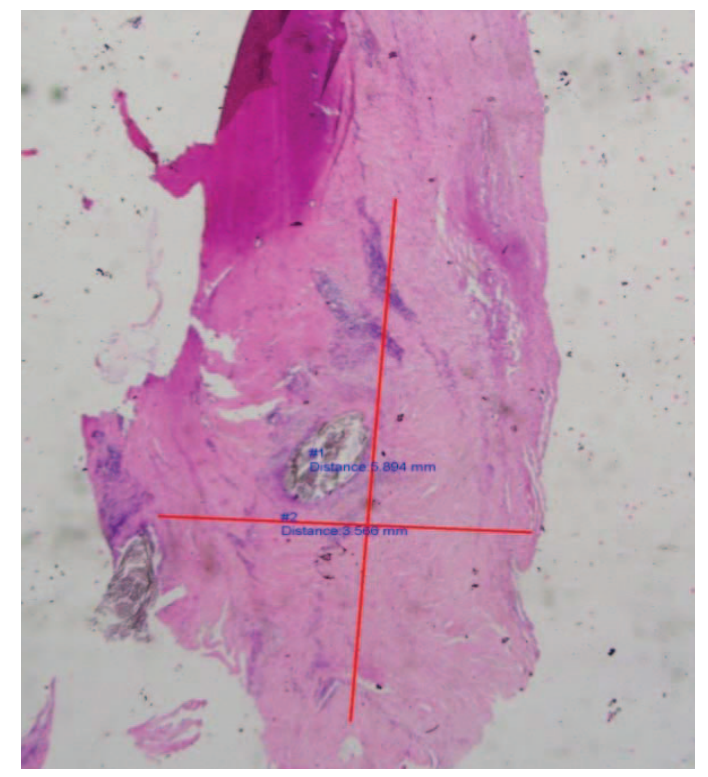

Fig 3. PA microscopic histological examination display with HE staining 40x magnification. Sample, obtained $5.89 \mathrm{~mm} \times 3.57 \mathrm{~mm}$ fibrocytes infiltration.

Amniotic membrane has tensile number $6.80 \pm 0.22 \mathrm{MPa}$ that makes it quite flexible [6]. Our results showed that samples with amniotic membrane graft treatment could be sutured along the edges of dural defect. This suturable characteristic would support the ideal properties of amniotic membrane as potential dura mater implant based on the mechanical properties.

Moreover, there was no CSF leakage around the dura mater graft during the clinical care and CSF fistula was not shown during treatment. This result also verified a previous study conducted by Tomita which is showed that the use of amniotic membrane graft in human subjects did not cause tissue rejection, infection or CSF leakage [5]. Without any leakage those samples would minimized CSS fistula, infection, herniation, adhesion of the cerebral cortex, and brain cortical scars.

Amniotic membrane showed a good biocompatibility by investigating the effect after implantation. There was no body rejection found in implant site since amniotic membrane was a biomaterial from human itself. This biocompatibility was related to the laminin-5 contained in the basement membrane of amnion. Amniotic membrane also found to be blended with surrounded tissue.

Based on the histological analysis, the growth of fibrositic infiltration began from the edge of the defect dura mater and muscle. Fibroblast cells fibrins bound form tightly supported by neovascularization in the area. Figure 3 showed that there was $5.89 \mathrm{~mm} \times 3.57 \mathrm{~mm}$ fibrocytes infiltration obtained. Those fibrocytes can initiate the regeneration of new dura mater tissue.

\section{CONCLUSION}

Based on the experiment it can be conclude that amniotic membrane graft was able to provide watertight effect while testing with $0.9 \% \mathrm{NaCl}$ injections. Amniotic membrane also showed capability of stimulating adequate fibrocyte infiltration based on the histological review, thus can support edge healing of the dura mater. This experiment can lead invention in the field of future implant technology.

\section{REFERENCES}

[1] Keller J, Ongkiko CM Jr, Saunders M. Repair of spinal dural defects. An experimental study. J Neurosurg 1984;60:1022-8.

[2] Simpson D, Robson A, 1984. Recurrent subarachnoid bleeding in association with dural substitute. Report of three cases. J Neurosurg 60:408-409

[3] Tomita T, Hayashi N. New dried human amniotic membrane Is useful as a substitute for dural repair after skull base surgery. J Neurol Surg B 2012;1:302-7.

[4] Thadani V, Penar PL, Partington J, Kalb R, Janssen R, Schonberger LB, et al. Creutzfeldt-Jakob disease probably acquired from a cadaveric dura mater graft. J Neurosurg 1988;69:766-9

[5] Tomita T, Hayashi N. New dried human amniotic membrane Is useful as a substitute for dural repair after skull base surgery. J Neurol Surg B 2012;1:302-7

[6] Tanaka K, T. Nagayama, T. Katayama, N. Koizumi. Tensile Properties of Amniotic Membrane. J. Tensile Poperties of Amniotic Membrane. WIT Transactions on the Built Environment. 112. 2010:197-206. 